Review Article

\title{
The Emerging Role of GC-MSCs in the Gastric Cancer Microenvironment: From Tumor to Tumor Immunity
}

\author{
Zhaoji Pan $\mathbb{D}^{1},{ }^{1}$ Yiqing Tian $\mathbb{D}^{2},{ }^{2}$ Guoping Niu $\mathbb{D}^{1},{ }^{1}$ and Chengsong Cao $\mathbb{D}^{1}$ \\ ${ }^{1}$ Xuzhou Central Hospital, The Affiliated Xuzhou Hospital of Medical College of Southeast University, Xuzhou, Jiangsu, China \\ ${ }^{2}$ Xinyi People's Hospital, Xinyi, Xuzhou, Jiangsu, China
}

Correspondence should be addressed to Yiqing Tian; 824303893@qq.com

Received 10 September 2019; Accepted 15 November 2019; Published 2 December 2019

Academic Editor: Kenichi Tamama

Copyright (c) 2019 Zhaoji Pan et al. This is an open access article distributed under the Creative Commons Attribution License, which permits unrestricted use, distribution, and reproduction in any medium, provided the original work is properly cited.

\begin{abstract}
Mesenchymal stem cells (MSCs) have been declared to not only participate in wound repair but also affect tumor progression. Tumor-associated MSCs, directly existing in the tumor microenvironment, play a critical role in tumor initiation, progression, and development. And different tumor-derived MSCs have their own unique characteristics. In this review, we mainly describe and discuss recent advances in our understanding of the emerging role of gastric cancer-derived MSC-like cells (GC-MSCs) in regulating gastric cancer progression and development, as well as the bidirectional influence between GC-MSCs and immune cells of the tumor microenvironment. Moreover, we also discuss the potential biomarker and therapeutic role of GC-MSCs. It is anticipated that new and deep insights into the functionality of GC-MSCs and the underlying mechanisms will promote the novel and promising therapeutic strategies against gastric cancer.
\end{abstract}

\section{Introduction}

Mesenchymal stem cells (MSCs), known as mesenchymal stromal cells as well, were first discovered in pig bone marrow stroma in 1960s and identified as fibroblast colonies [1]. Then, they were found to exist in most tissues and play significantly important roles in several types of disease, including inflammatory diseases, tissue regeneration healing, and organ injury diseases [2-11]. MSCs have the plasticity characteristic, which means they could not only enhance tissue healing and promote immune responses but also have the inhibitory function, according to the pathophysiological status of the tissue where they reside $[12,13]$. Recently, MSCs have been found to affect tumor progression and function as key regulators of tumor fate $[9,14-17]$. And MSCs derived from different tumor types could influence tumor progression through different mechanisms. Tumor-associated MSCs (TA-MSCs) from ovarian cancer or multiple myeloma were reported to promote tumor growth by secreting some growth factors or exosomes $[18,19]$. In a human colorectal cancer xenograft model, TA-MSCs could promote tumor angiogenesis in an IL-6- and endothelin-1-dependent way, whereas
CAFs and normal fibroblasts could not [20]. Moreover, TA-MSCs in breast cancer enhanced the motility, invasive ability, and metastasis of tumor cells by CCL5/CCR5 signaling axis [21]. So, TA-MSCs are distinctively unique in different tumor types.

The tumor microenvironment (TME) is the complex microenvironment composed of different cellular types including tumor cells, endothelial cells, stromal cells, and immune cells $[22,23]$. Tumors are considered to be wounds which do not heal [24], and MSCs were reported to have the immunosuppressive functionality [25]. Recently, many studies have demonstrated that MSCs could affect the phenotype and functionality of $\mathrm{T}$ cells including mediating the $\mathrm{CD} 4^{+} \mathrm{T}$ cell migration and differentiation [26], modulating $\mathrm{T}$ helper 17/regulatory T balance [27], and controlling memory T cell responses [28]. MSCs are also involved in the immunomodulatory function of B cells, dendritic cells, macrophages, and myeloid-derived suppressor cells (MDSCs) [29-32]. So, it is easily understandable that MSCs interact with immune cells and other cells in the TME. Moreover, MSCs have been reported to influence tumor progression through regulating immune cells in different tumor types [33-36]. However, 
studies about the tumor immunity role of TA-MSCs are still in infancy. Gastric cancer, the leading cause of cancer-related death worldwide, is highly concerned [37-41]. Emerging evidence demonstrated that the tumor microenvironment cells including macrophages, $\mathrm{T}$ cells, and fibroblasts all play critical roles in GC development and prognosis [42-45]. In this review, we mainly detail and discuss current advances in the understanding of the important role of gastric cancerderived MSC-like cells (GC-MSCs) in gastric cancer (GC) progression. We would elaborate from how GC-MSCs interact with tumor cells to interacting with immune cells and how their interactions impact tumor progression, which is greatly meaningful for gastric cancer immunotherapy.

\section{GC-MSCs}

2.1. The Origin of GC-MSCs. In 2004, Studeny et al. found that bone-marrow-derived MSCs (BM-MSCs) could recruit to tumors after the intravenous injection of MSCs [46], which laid the foundation for later MSC-associated studies. In 2012, Ren et al. furtherly verified that the intrabone injection-derived green fluorescent protein $(\mathrm{GFP})^{+} \mathrm{BM}-$ MSCs could actively recruit to tumors [47]. Surprisingly, they also proved that tumor-resident MSCs are derived from BM-MSCs, revealing that BMMSCs maybe the precursors of TA-MSCs. In 2014, Ren et al. continue to demonstrate that lymphoma-resident MSCs endowed BM-MSCs with tumor-promoting properties [48], indicating that TAMSCs could transfer BM-MSCs into TA-MSCs to expand their numbers. Supplementally, miR-155-5p inhibition was proved to promote the transition of BM-MSC into GCMSC by targeting NF- $\kappa \mathrm{B}$ p65, in 2016 [49]. To sum up, BM-MSCs are the main origin of GC-MSCs, which is greatly critical for GC progression.

2.2. Isolation and Characteristics. In 2008, GC-MSCs, the main component of the TME of GC, were firstly discovered and isolated by Cao et al. [50]. Probably 15 days after plating, GC-MSCs commenced to form colonies, with long and spindle-shaped single cells, resembling the morphology of fibroblasts. The GC-MSCs also had a normal number of chromosomes that could maintain the shape and structure of MSCs after cell cycle progression. Moreover, contrary to GC cells, the GC-MSCs failed to form tumors when grafted into nude mice, indicating that GC-MSCs were normal cells but not a subset of tumor cells. GC-MSCs express stem cellrelated genes including Oct-4, CD44, CD73, Nanog, Bmil, and nucleostemin but were negative for hematopoietic markers such as CD34 and were negative for the specific endothelial antigen, CD31. And it has the ability to selfrenew and differentiate into osteocytes or adipocytes, which was similar with BM-MSCs.

So far, the uniform standard for the biological characteristics and function of TA-MSCs is rare. In different tumor types, TA-MSCs were proved different new characteristics that were significantly different from BM-MSCs. In human ovarian cancers, TA-MSCs secrete obviously more bone morphogenetic proteins, which are indispensable for maintaining stem cell differentiation, than BM-MSCs [18]. In multiple myeloma, compared with BM-MSCs, TA-MSCs express lower levels of miR-15a, the tumor-suppressive microRNA (miRNA) [19]. In gastric cancer, Cao et al. demonstrated that GC-MSCs have higher proliferative potential than BM-MSCs, endowing GC-MSCs with quicker selfrenew ability, which is consistent with the high expression of PCNA in GC-MSCs (most of BM-MSCs were negative for PCNA expression) [50].

In 2010, Xu et al. found and isolated the human gastric cancer adjacent noncancerous tissue-derived MSCs (hGCNMSCs), which are paired with the GC-MSCs [51]. hGCNMSCs and GC-MSCs are alike in the typical fibroblast-like appearance, paralleling to that of hBM-MSCs. In addition, both hGCN-MSCs and GC-MSCs were positive for CD29, CD44, CD90, and CD105, but negative for CD14, CD31, $\mathrm{CD} 34$, and CD45, which were the characteristic surface of MSCs. However, differences exist between them. The cumulative population of GC-MSCs is doubling the hGCN-MSCs and BM-MSCs. Compared with hGCN-MSCs, GC-MSCs showed a lower migration ability and CD44 expression, which is one of the important adhesion molecules that are responsible for cell migration or invasion [52].

\section{GC-MSCs and Tumor}

The following part will detail the interaction between GCMSCs and gastric cancer environment, which plays a significantly important role in tumor development.

3.1. GC-MSC Secreted Molecules and Tumor Progression. Huang et al. found that PDGF-DD from the GC-MSCconditioned medium (CM) could promote the proliferation and migration of tumor cells in vitro and in vivo by phosphorylating PDGFR- $\beta$ in SGC-7901 cells [53]. And targeting the PDGF-DD/PDGFR- $\beta$ interaction between GC-MSCs and tumor cells may provide a novel strategy for gastric cancer therapy. However, whether a molecule or a signaling pathway in GC-MSCs or other microenvironmental cells regulate the secretion of PDGF-DD were still unknown, which need to be further investigated. Moreover, another proinflammatory cytokine which was strikingly high secreted by GC-MSCs, interleukin-8 (IL-8), was reported to enhance the proliferation, migration, and proangiogenesis ability of GC cells partly by regulating the activation of Akt or Erk1/2 pathway, which also participated in immune regulation we would elucidate in the following tumor immunity part [54]. Either knocking down PDGF-DD in GC-MSCs or adding anti-IL-8 antibody reversed the tumor promoting role of hGC-MSCs in vitro and in vivo. However, whether the signaling pathways PDGF-DD and IL-8 participate in crossed connection need to be investigated. Sun et al. also demonstrated that GC-MSC-derived IL-15 could promote GC cell migration and epithelial-mesenchymal transition (EMT) by regulating STAT3 in GC cells [55]. Above all, GC-MSCs could secrete some molecules to promote tumor progression.

3.2. GC-MSC Expressed Molecules and Tumor Progression. miRNAs, the short noncoding RNAs, regulating posttranscriptional gene expression, act as oncogenes or tumor 
suppressors that are involved in tumor progression [56-58]. The role of miRNAs in TA-MSCs catches more and more attention, which is crucial for tumor progression [59-61]. Wang et al. firstly identified that miR-214, miR-221, and miR-222 are upregulated in GC-MSCs and gastric cancer tissues compared to GC-MSCs and adjacent noncancerous tissues [62]. Moreover, they also found that miR-221 inhibiting in GC-MSCs could obviously suppress the proliferation and migration of GC cells. Mechanistically, GCMSC-CM-derived exosome carrying miR-221 could be delivered to GC cells to enhance the miR-221 expression in GC cells, which regulate the proliferative and migratory ability of GC cells. In addition, miR-374 in GC-MSCs, which were isolated from $\mathrm{N}$-methyl- ${ }^{\prime}$-nitro- $\mathrm{N}$-nitrosoguanidine(MNNG-) induced gastric cancer from gastritis in a rat experimental model, regulated their proliferative and migratory capabilities, which is critical for gastric carcinogenesis [63]. Zhu et al. not only found that miR-155-5p inhibition could regulate the transition of BM-MSC into GC-MSClike cells acquiring a GC-MSC-like phenotype and function but also proved that miR-155-5p overexpression in GCMSCs inhibited growth, migration, and invasion of GC in vitro and in vivo [49]. Those results show that miRNA in GC-MSCs is greatly important for GC progression and development.

In addition to miRNA, other molecules expressed in GCMSCs also can regulate GC progression. We have proved that YAP expression in GC-MSCs can affect GC growth and progression in vitro and in vivo. However, the detailed mechanisms are not described [64]. Whether YAP regulate the molecule secretion in the GC-MSC-CM or regulate other microenvironmental cells to influence the GC progression and what is the downstream signaling pathway in GC cells are still needed to be further investigated.

Above all, molecules of GC-MSCs secreting and expressing could influence GC progression by different mechanisms.

\section{GC-MSCs and Tumor Immunity}

Accumulating evidence has demonstrated that TA-MSCs could affect tumor progression through the bidirectional interactions with innate and adaptive immune systems [34, $47,65,66]$. Herein, we highlight the interactions between GC-MSCs and immune cells or molecules, and how they impact tumor progression, which are very interesting and meaningful for GC immunotherapy.

4.1. GC-MSCs and Innate Immunity. TA-MSCs have been reported to recruit innate immune cells, macrophages, and neutrophils in particular and interact with them to enhance the anti-inflammatory condition, thereby promoting tumor progression [23, 67-70]. Based on studies to date, there are few to no studies about the interaction between GC-MSCs and macrophages or myeloid-derived suppressor cells (MDSCs). Here, we mainly elucidate the communication between GC-MSCs and neutrophils. Zhu et al. first proved that bidirectional interactions between GC-MSCs and neutrophils exist in the GC environment, which could influence the GC progression [71]. They con- cluded that GC-MSC-CM remarkably prompted the chemotaxis of neutrophils and skewed them towards the activated state. Moreover, GC-MSC-CM could also inhibit the spontaneous apoptosis of neutrophils. Although GCMSC-primed neutrophils have no function on GC cell proliferation, they promote the migration and angiogenesis of GC cells and could induce the transition of GCN-MSCs into CAFs. TA-MSCs were reported to have the ability to differentiate into $\alpha$-SMA ${ }^{+}$CAFs [72, 73]. And whether GC-MSC-primed neutrophils are able to induce GCMSCs into CAFs, which also have a tumor-promoting role, need to be further investigated. Mechanistically, GC-MSC-CM-derived IL-6 mediated the activation of STAT3-ERK1/2 signaling cascade in neutrophils, regulating the above communication between GC-MSCs and neutrophils, and their impacts on the promigratory and proangiogenicity of GC cells. These results show the importance of bidirectional interaction between GCMSCs and innate immunity, which could obviously influence tumor progression.

4.2. GC-MSCs and Adaptive Immunity. Emerging evidence has demonstrated that MSCs have immunosuppressive effects on the adaptive immune system, thereby enhancing tumor growth [74-76]. TA-MSCs are the crucial component of the TME and are more direct than BM-MSCs in the study of interactions among MSCs, immune cells, tumor cells, and other environmental cells. Wang et al. found that GC-MSC-CM could obviously reverse the inhibitory effects of peripheral blood mononuclear cells (PBMCs) on the GC growth in vivo, but not in vitro [77]. And GC-MSC-CM-educated PBMCs could significantly enhance the migration and EMT of GC cells in vitro and liver metastases in vivo. Moreover, GCMSC-CM dampened Treg/Th17 balance in PBMCs through inhibiting Th17 cell proliferation and inducing Treg differentiation. Mechanistically, Sun et al. further proved that GC-MSC-derived IL-15 increased Tregs by activating STAT5 in $\mathrm{CD}^{+}{ }^{+} \mathrm{T}$ cells, which enhanced GC cell migration [55]. Unexpectedly, they found that IL-15 also promoted PD-1 expression in Tregs, which was worth further investigating [55]. Interestingly, GC-MSC-derived IL-8 could regulate immune checkpoints, PD-L1 in GC cells through STAT3/mTOR-c-Myc signal axis [78]. And it also protects GC cells from the cytotoxic effect of $\mathrm{CD}^{+} \mathrm{T}$ cells by upregulating PD-L1 in GC cells [78]. As shown above, GC-MSCs can regulate the functionality of adaptive immune cells, thereby influencing tumor progression. Xu et al. proved that GC-MSCs are plastic, whose phenotype and immunomodulatory ability could be modified by adaptive immune cells [79]. $\mathrm{CD}^{+} \mathrm{T}$ cells could educate GC-MSCs with PD-L1 upregulation, which promoted GC cell migration and GC growth in vivo via PD-1/mammalian target of rapamycin (mTOR) signaling axis while did not impact apoptosis and proliferation of GC cells in vitro [79]. To sum up, bidirectional interactions between GC-MSCs and adaptive system cells greatly affect the GC progression, which may provide novel approaches for immunotherapy. 


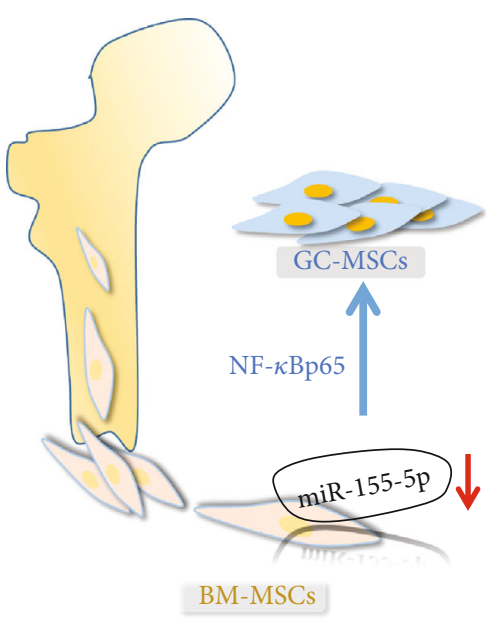

(a)

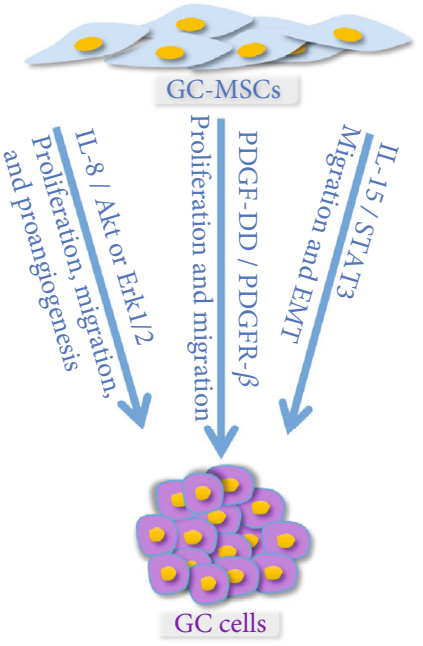

(b)

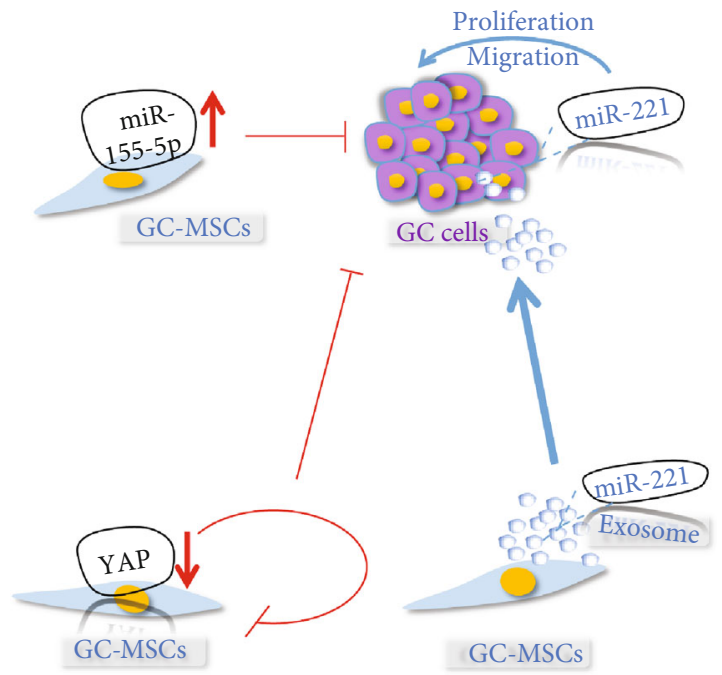

(c)

Figure 1: The important role of GC-MSCs in GC progression. (a) GC-MSC origin from BM-MSCs, which could be mediated by miR-155-5p inhibition to transfer into GC-MSCs. (b) GC-MSC-derived CM, containing molecules including IL-8, IL-15, and PDGF-DD, promote GC progression through AKT/ERK, PDGF-DD/PDGFR- $\beta$, IL-15/STAT3 signaling pathways, respectively. (c) Changes of molecule expression in GC-MSC influence GC progression. miR-155-5p overexpression in GC-MSCs could reverse the tumor-promoting phenotype and function; YAP knockdown in GC-MSCs inhibits GC-MSC functionality and suppresses the GC growth; miR-221 in GC-MSCs could be delivered to GC cells through exosomes, which increase miR-21 expression in GC cells and promote GC progression.

\section{Biomarker and Therapy}

Emerging studies have shed light on the diagnostic and prognostic potential of tumoral miRNAs in GC [80, 81]. Deregulated miRNA levels have been investigated clinically acting as diagnostic biomarkers in several biopsy specimens and body fluids $[82,83]$. But few studies demonstrated the diagnostic and prognostic value of miRNAs in TA-MSCs. Wang et al. found that miR-214 in circulating levels and miR-221 and miR-222 in GC-MSC levels were significantly higher in the GC-MSC group than in the relevant GCN-MSC group, which was consistent with the differential expression levels between GC tissues and noncancerous gastric tissues [62]. They also proved the correlations, respectively, between miR-221 and miR-222 high levels and extensive lymph node metastasis, miR-214 and miR-222 high levels and serosal invasion, miR-221 and miR-222 high levels and the TNM stage, and miR-214 high levels and venous invasion [62]. In addition to miRNA, other molecules from GC-MSCs have the potential to act as a biomarker. The elevated serum IL15, which was also mainly derived from GC-MSCs, was proved to be significantly correlated with lymph node metastasis in GC, but not with other clinicopathological parameters [55]. To summarize, GC-MSCs also have the diagnostic and prognostic value, which is crucial for clinical therapy.

Studies of the GC-MSC-associated therapy are rare, which means more efforts need to be made in this field. Huang et al. found a compound, named curcumin, suppressing GC-MSC-mediated angiogenesis through inhibiting NF- $\kappa$ B/VEGF signaling axis, which means a novel therapeutic approach for GC progression via targeting GCMSC-driven angiogenesis [84]. Interestingly, we also discerned that low level 3,3'-diindolylmethane (DIM) evidently enhanced GC cell proliferation and migration through activating wnt4 signaling while high level obviously inhibited GC growth, which provide a new strategy for the optimum concentration of DIM in its clinical application. Also, the effects of DIM on the functions of GC-MSCs need to be further investigated [85].

In summary, GC-MSCs could play the important biomarker and therapeutic role in GC progression, providing potentially novel therapeutic targets for tumor therapy.

\section{Discussion and Perspectives}

Diverse potential abilities of MSCs are constantly being excavated, from its crucial role in protumor and antitumor progression to recently several novel MSC-mediated therapies [73, 86-90]. TA-MSCs, as the critical component of TME, are concerned by more and more studies. They interact with microenvironmental cells more directly than other MSC type, so it is meaningful to deeply investigate the further function of TA-MSCs. Tumor immunity could obviously influence tumor fate. It is easier to combine with the clinic and has a better clinical application value, which is a well-being of tumor treatment [91-95]. So, correlations between TA-MSCs and tumor immunity need to be given more attention and investigated, which is promising and could offer a blueprint for tumor immunotherapy. In this review, we mainly elucidate the essential and indispensable role of GC-MSCs in the GC progression from tumor to tumor immunity, which has the potential therapeutic value (Figures 1 and 2, Table 1). And thought-provoking in the above GC-associated studies, we would discuss here. PD-1 and PD-L1 pair, the leading immune checkpoint pathway in the TME, plays a immunosuppressive role by suppressing 


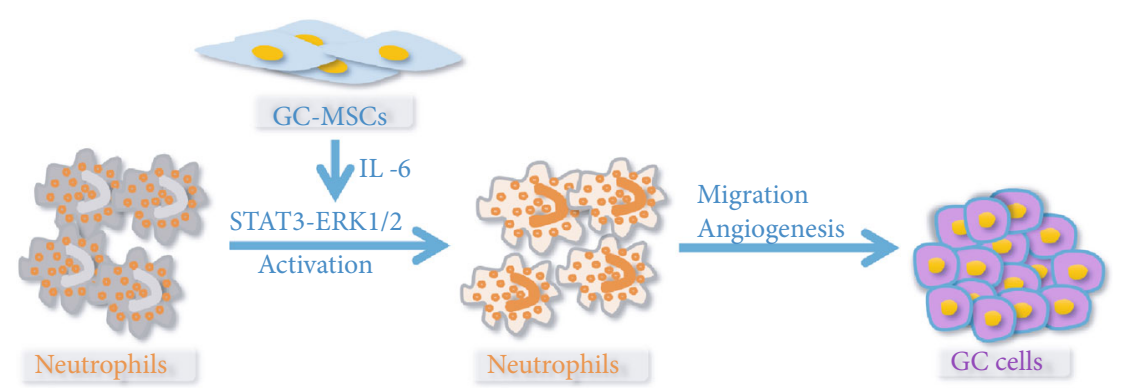

(a)

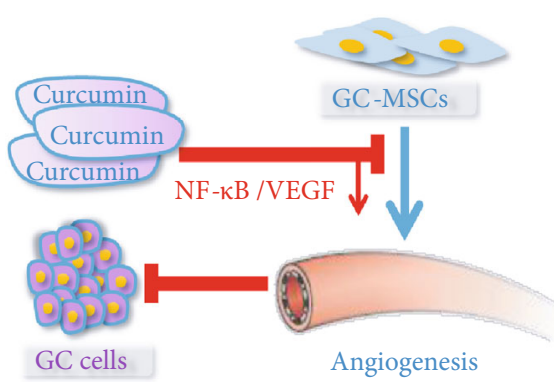

(c)

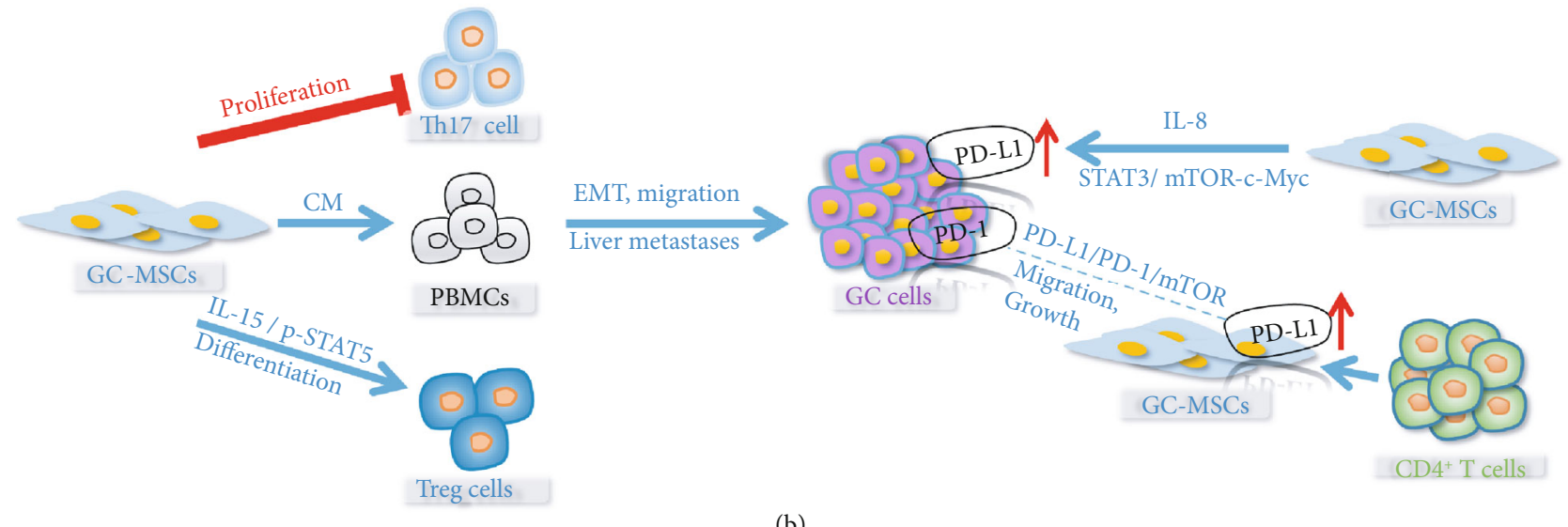

(b)

FIGURE 2: The interaction between GC-MSCs and immune cells and therapy. (a) GC-MSC-derived IL-6 induces the activation of neutrophils through STAT3-ERK1/2 signaling axis, promoting migration and angiogenesis of GC cells. (b) GC-MSC-CM inhibits the proliferation of antitumor immune cells, Th17 cells. GC-MSC-derived IL-15 can promote the differentiation of protumor immune cells, Tregs through activating STAT5. GC-MSC-educated PBMCs could also induce migration and the EMT of GC cells. GC-MSC-derived IL-8 could promote PD-L1 expression in GC cells through STAT3/mTOR-c-Myc signaling axis. CD4 ${ }^{+} \mathrm{T}$ cells educate GC-MSCs with PD-L1 upregulation, promoting GC cell migration and GC growth via activating PD-1/mTOR signaling. (c) Curcumin could inhibit GC-MSCmediated angiogenesis and suppress GC growth.

TABLE 1: The emerging role of GC-MSCs in the gastric cancer microenvironment.

\begin{tabular}{|c|c|c|}
\hline Critical molecules/cells & Effect & Reference \\
\hline PDGF-DD & $\begin{array}{l}\text { GC-MSC-derived PDGF-DD promoted GC cell proliferation and } \\
\text { migration by PDGF-DD/PDGFR- } \beta \text { signaling pathway. }\end{array}$ & {$[53]$} \\
\hline IL-8 & $\begin{array}{l}\text { GC-MSC-derived IL- } 8 \text { enhanced the proliferation, migration, and proangiogenesis } \\
\text { ability of GC cells partly by regulating the activation of Akt or Erk1/2 pathway. }\end{array}$ & {$[54]$} \\
\hline IL-15 & $\begin{array}{l}\text { GC-MSC-derived IL-15 could promote GC cell migration and epithelial-mesenchymal } \\
\text { transition (EMT) by regulating STAT3 in GC cells. }\end{array}$ & {$[55]$} \\
\hline miR-221 & $\begin{array}{l}\text { GC-MSC-CM-derived exosome carrying miR-221 regulated the proliferative } \\
\text { and migratory ability of GC cells. }\end{array}$ & {$[62]$} \\
\hline $\operatorname{miR}-374$ & miR-374 participated in regulating gastric carcinogenesis. & [63] \\
\hline miR-155-5p & miR-155-5p inhibition could regulate the transition of BM-MSC into GC-MSC-like cells. & [49] \\
\hline YAP & YAP expression in GC-MSCs can affect GC growth and progression in vitro and in vivo. & [64] \\
\hline Neutrophils & $\begin{array}{l}\text { GC-MSC-CM remarkably prompted the chemotaxis of neutrophils and skewed them towards } \\
\text { the activated state through GC-MSC-CM-derived IL- } 6 \text { that mediated the activation of STAT3- } \\
\text { ERK1/2 signaling in neutrophils, which could promote migration and angiogenesis of GC. }\end{array}$ & [71] \\
\hline PBMCs & $\begin{array}{l}\text { GC-MSC-CM could obviously reverse the inhibitory effects of peripheral blood mononuclear cells } \\
\text { (PBMCs) on the GC growth. And GC-MSC-CM dampened Treg/Th17 balance in PBMCs. }\end{array}$ & {$[77]$} \\
\hline
\end{tabular}

the function of $\mathrm{T}$ cells and tumor-infiltrating lymphocytes, resultingly promoting tumor progression [96, 97]. Owing to the role of GC-MSC-derived IL- 8 and the function of
$\mathrm{CD}^{+} \mathrm{T}$ cell-primed GC-MSCs with PD-L1 upregulation we have described [54, 78, 79], we speculate that PD-L1 expression in GC-MSCs could affect IL- 8 secretion and 
mediate the GC progression, which we are investigating recently. GC-MSC-CM, CD4 ${ }^{+} \mathrm{T}$ cell-primed GC-MSC-CM and GC-MSC-primed neutrophils, had no impact on the proliferation of GC cells in vitro, but all of them promote GC growth in vivo $[71,79]$, which remind that the underlying microenvironmental cells or mechanisms work. Whether the proangiogenic role of GC-MSCs only works in vivo need to be further investigated [98]. Moreover, the interactions between GC-MSCs and other immune cells including B cells, macrophages, NK cells, and MDSCs need to be further investigated, which are more valuable for deeply exploring the correlation between GC and immune systems and the effectively clinical immunotherapy.

\section{Conflicts of Interest}

All authors have no conflicts of interest relevant to this article.

\section{Authors' Contributions}

Zhaoji Pan and Yiqing Tian contributed equally to this manuscript.

\section{Acknowledgments}

We would like to thank Professor Hui Qian and the Key Laboratory of Laboratory Medicine of Jiangsu Province, School of Medicine, Jiangsu University, Zhenjiang, Jiangsu, P. R. China, for their contribution on this article. The study was financially supported by the Special Foundation for Young Scientists of Jiangsu Province (Grant no. QNRC2016379), Xuzhou Central Hospital, and Xinyi People's Hospital.

\section{References}

[1] A. J. Friedenstein, R. K. Chailakhjan, and K. S. Lalykina, “The development of fibroblast colonies in monolayer cultures of guinea-pig bone marrow and spleen cells," Cell and Tissue Kinetics, vol. 3, no. 4, pp. 393-403, 1970.

[2] A. Uccelli, L. Moretta, and V. Pistoia, "Mesenchymal stem cells in health and disease," Nature Reviews. Immunology, vol. 8, no. 9, pp. 726-736, 2008.

[3] S. Y. Min, A. Desai, Z. Yang et al., "Diverse repertoire of human adipocyte subtypes develops from transcriptionally distinct mesenchymal progenitor cells," Proceedings of the National Academy of Sciences of the United States of America, vol. 116, no. 36, pp. 17970-17979, 2019.

[4] M. E. Bernardo and W. E. Fibbe, "Mesenchymal stromal cells: sensors and switchers of inflammation," Cell Stem Cell, vol. 13, no. 4, pp. 392-402, 2013.

[5] S. M. Haikal, N. F. Abdeltawab, L. A. Rashed, T. I. Abd el-Galil, H. A. Elmalt, and M. A. Amin, "Combination therapy of mesenchymal stromal cells and interleukin- 4 attenuates rheumatoid arthritis in a collagen-induced murine model," Cell, vol. 8, no. 8, p. 823, 2019.

[6] B. Zhang, Y. Shi, A. Gong et al., "HucMSC exosome-delivered 14-3-3 $\zeta$ orchestrates self-control of the Wnt response via modulation of YAP during cutaneous regeneration," Stem Cells, vol. 34, no. 10, pp. 2485-2500, 2016.
[7] K. Pan, L. Deng, P. Chen et al., "Safety and Feasibility of Repeated Intrathecal Allogeneic Bone Marrow- Derived Mesenchymal Stromal Cells in Patients with Neurological Diseases," Stem Cells International, vol. 2019, Article ID 8421281, 15 pages, 2019.

[8] M. Carvello, A. Lightner, T. Yamamoto, P. G. Kotze, and A. Spinelli, "Mesenchymal stem cells for perianal Crohn's disease," Cell, vol. 8, no. 7, p. 764, 2019.

[9] S. M. Ridge, F. J. Sullivan, and S. A. Glynn, "Mesenchymal stem cells: key players in cancer progression," Molecular Cancer, vol. 16, no. 1, p. 31, 2017.

[10] K. Le Blanc, I. Rasmusson, B. Sundberg et al., "Treatment of severe acute graft-versus-host disease with third party haploidentical mesenchymal stem cells," The Lancet, vol. 363, no. 9419, pp. 1439-1441, 2004.

[11] Y. Han, X. Li, Y. Zhang, Y. Han, F. Chang, and J. Ding, "Mesenchymal stem cells for regenerative medicine," Cell, vol. 8, no. 8, p. 886, 2019.

[12] M. S. Hu, M. R. Borrelli, H. P. Lorenz, M. T. Longaker, and D. C. Wan, "Mesenchymal stromal cells and cutaneous wound healing: a comprehensive review of the background, role, and therapeutic potential," Stem Cells International, vol. 2018, Article ID 6901983, 13 pages, 2018.

[13] Y. Wang, X. Chen, W. Cao, and Y. Shi, "Plasticity of mesenchymal stem cells in immunomodulation: pathological and therapeutic implications," Nature Immunology, vol. 15, no. 11, pp. 1009-1016, 2014.

[14] B. M. Beckermann, G. Kallifatidis, A. Groth et al., "VEGF expression by mesenchymal stem cells contributes to angiogenesis in pancreatic carcinoma," British Journal of Cancer, vol. 99, no. 4, pp. 622-631, 2008.

[15] A. De Boeck, P. Pauwels, K. Hensen et al., "Bone marrowderived mesenchymal stem cells promote colorectal cancer progression through paracrine neuregulin 1/HER3 signalling," Gut, vol. 62, no. 4, pp. 550-560, 2013.

[16] M. Timaner, K. K. Tsai, and Y. Shaked, "The multifaceted role of mesenchymal stem cells in cancer," Seminars in Cancer Biology, 2019.

[17] C. Melzer, Y. Yang, and R. Hass, "Interaction of MSC with tumor cells," Cell Communication and Signaling: CCS, vol. 14, no. 1, p. 20, 2016.

[18] K. McLean, Y. Gong, Y. Choi et al., "Human ovarian carcinoma-associated mesenchymal stem cells regulate cancer stem cells and tumorigenesis via altered BMP production," The Journal of Clinical Investigation, vol. 121, no. 8, pp. 3206-3219, 2011.

[19] A. M. Roccaro, A. Sacco, P. Maiso et al., "BM mesenchymal stromal cell-derived exosomes facilitate multiple myeloma progression," The Journal of Clinical Investigation, vol. 123, no. 4, pp. 1542-1555, 2013.

[20] W. H. Huang, M. C. Chang, K. S. Tsai, M. C. Hung, H. L. Chen, and S. C. Hung, "Mesenchymal stem cells promote growth and angiogenesis of tumors in mice," Oncogene, vol. 32 , no. 37, pp. 4343-4354, 2013.

[21] A. E. Karnoub, A. B. Dash, A. P. Vo et al., "Mesenchymal stem cells within tumour stroma promote breast cancer metastasis," Nature, vol. 449, no. 7162, pp. 557-563, 2007.

[22] G. U. Dachs and D. J. Chaplin, "Microenvironmental control of gene expression: implications for tumor angiogenesis, progression, and metastasis," Seminars in Radiation Oncology, vol. 8, no. 3, pp. 208-216, 1998. 
[23] D. F. Quail and J. A. Joyce, "Microenvironmental regulation of tumor progression and metastasis," Nature Medicine, vol. 19, no. 11, pp. 1423-1437, 2013.

[24] H. F. Dvorak, "Tumors: wounds that do not heal. Similarities between tumor stroma generation and wound healing," The New England Journal of Medicine, vol. 315, no. 26, pp. 16501659, 1986.

[25] M. P. De Miguel, S. Fuentes-Julian, A. Blazquez-Martinez et al., "Immunosuppressive properties of mesenchymal stem cells: advances and applications," Current Molecular Medicine, vol. 12, no. 5, pp. 574-591, 2012.

[26] S. Cen, P. Wang, Z. Xie et al., "Autophagy enhances mesenchymal stem cell-mediated $\mathrm{CD} 4^{+} \mathrm{T}$ cell migration and differentiation through CXCL8 and TGF- $\beta 1$," Stem Cell Research \& Therapy, vol. 10, no. 1, p. 265, 2019.

[27] K. Tumangelova-Yuzeir, E. Naydenov, E. Ivanova-Todorova et al., "Mesenchymal stem cells derived and cultured from glioblastoma multiforme increase Tregs, downregulate Th17, and induce the tolerogenic phenotype of monocyte-derived cells," Stem Cells International, vol. 2019, Article ID 6904638, 15 pages, 2019.

[28] N. Luque-Campos, R. A. Contreras-Lopez, M. Jose ParedesMartinez et al., "Mesenchymal stem cells improve rheumatoid arthritis progression by controlling memory T cell response," Frontiers in Immunology, vol. 10, p. 798, 2019.

[29] X. Chen, C. Cai, D. Xu et al., "Human mesenchymal stem celltreated regulatory $\mathrm{CD} 23^{+} \mathrm{CD} 43^{+} \mathrm{B}$ cells alleviate intestinal inflammation," Theranostics, vol. 9, no. 16, pp. 4633-4647, 2019.

[30] T. Ghosh, S. Barik, A. Bhuniya et al., "Tumor-associated mesenchymal stem cells inhibit naïve T cell expansion by blocking cysteine export from dendritic cells," International Journal of Cancer, vol. 139, no. 9, pp. 2068-2081, 2016.

[31] T. Lin, J. Pajarinen, Y. Kohno et al., “Trained murine mesenchymal stem cells have anti-inflammatory effect on macrophages, but defective regulation on T-cell proliferation," The FASEB Journal, vol. 33, no. 3, pp. 4203-4211, 2019.

[32] H. J. Lee, J. H. Ko, H. J. Jeong et al., "Mesenchymal stem/stromal cells protect against autoimmunity via CCL2-dependent recruitment of myeloid-derived suppressor cells," Journal of Immunology, vol. 194, no. 8, pp. 3634-3645, 2015.

[33] R. J. Tang, S. N. Shen, X. Y. Zhao et al., "Mesenchymal stem cells-regulated Treg cells suppress colitis-associated colorectal cancer," Stem Cell Research \& Therapy, vol. 6, no. 1, p. 71, 2015.

[34] S. A. Patel, J. R. Meyer, S. J. Greco, K. E. Corcoran, M. Bryan, and P. Rameshwar, "Mesenchymal stem cells protect breast cancer cells through regulatory $\mathrm{T}$ cells: role of mesenchymal stem cell-derived TGF- $\beta$," Journal of Immunology, vol. 184, no. 10, pp. 5885-5894, 2010.

[35] Y. Yang, X. Zhang, F. Lin et al., "Bispecific CD3-HAC carried by E1A-engineered mesenchymal stromal cells against metastatic breast cancer by blocking PD-L1 and activating T cells," Journal of Hematology \& Oncology, vol. 12, no. 1, p. 46, 2019.

[36] F. Liotta, V. Querci, G. Mannelli et al., "Mesenchymal stem cells are enriched in head neck squamous cell carcinoma, correlates with tumour size and inhibit T-cell proliferation," British Journal of Cancer, vol. 112, no. 4, pp. 745-754, 2015.

[37] R. Li, J. Jiang, H. Shi, H. Qian, X. Zhang, and W. Xu, "CircRNA: a rising star in gastric cancer," Cellular and Molecular Life Sciences, pp. 1-20, 2019.
[38] X. Z. Dong, Z. R. Zhao, Y. Hu, Y. P. Lu, P. Liu, and L. Zhang, "LncRNA COL1A1-014 is involved in the progression of gastric cancer via regulating CXCL12-CXCR4 axis," Gastric Cancer, pp. 1-13, 2019.

[39] C. Wen, H. Wang, X. Wu et al., "ROS-mediated inactivation of the PI3K/AKT pathway is involved in the antigastric cancer effects of thioredoxin reductase-1 inhibitor chaetocin," Cell Death \& Disease, vol. 10, no. 11, p. 809, 2019.

[40] Y. Wei, F. Zhang, T. Zhang et al., "LDLRAD2 overexpression predicts poor prognosis and promotes metastasis by activating $\mathrm{Wnt} / \beta$-catenin/EMT signaling cascade in gastric cancer," Aging (Albany NY), vol. 11, no. 20, pp. 8951-8968, 2019.

[41] B. Yue, C. Song, L. Yang et al., "METTL3-mediated N6methyladenosine modification is critical for epithelialmesenchymal transition and metastasis of gastric cancer," Molecular Cancer, vol. 18, no. 1, p. 142, 2019.

[42] T. Li, B. Li, A. Sara et al., "Docking protein-1 promotes inflammatory macrophage signaling in gastric cancer," Oncoimmunology, vol. 8, no. 11, article e1649961, 2019.

[43] Q. Zhou, X. Wu, X. Wang et al., "The reciprocal interaction between tumor cells and activated fibroblasts mediated by TNF- $\alpha /$ IL-33/ST2L signaling promotes gastric cancer metastasis," Oncogene, 2019.

[44] F. Li, Y. Sun, J. Huang, W. Xu, J. Liu, and Z. Yuan, "CD4/CD8 ${ }^{+}$ T cells, DC subsets, Foxp 3, and IDO expression are predictive indictors of gastric cancer prognosis," Cancer Medicine, 2019.

[45] C. Kuo, C. Y. Chen, H. R. Lo et al., "Helicobacter pylori induces IL-33 production and recruits ST-2 to lipid rafts to exacerbate inflammation," Cell, vol. 8, no. 10, article 1290, 2019.

[46] M. Studeny, F. C. Marini, J. L. Dembinski et al., "Mesenchymal stem cells: potential precursors for tumor stroma and targeteddelivery vehicles for anticancer agents," Journal of the National Cancer Institute, vol. 96, no. 21, pp. 1593-1603, 2004.

[47] G. Ren, X. Zhao, Y. Wang et al., "CCR2-dependent recruitment of macrophages by tumor-educated mesenchymal stromal cells promotes tumor development and is mimicked by TNF $\alpha$," Cell Stem Cell, vol. 11, no. 6, pp. 812-824, 2012.

[48] G. Ren, Y. Liu, X. Zhao et al., "Tumor resident mesenchymal stromal cells endow naive stromal cells with tumorpromoting properties," Oncogene, vol. 33, no. 30, pp. 40164020, 2014.

[49] M. Zhu, M. Wang, F. Yang et al., "miR-155-5p inhibition promotes the transition of bone marrow mesenchymal stem cells to gastric cancer tissue derived MSC-like cells via NF- $\kappa \mathrm{B}$ p65 activation," Oncotarget, vol. 7, no. 13, pp. 16567-16580, 2016.

[50] H. Cao, W. Xu, H. Qian et al., "Mesenchymal stem cell-like cells derived from human gastric cancer tissues," Cancer Letters, vol. 274, no. 1, pp. 61-71, 2009.

[51] X. Xu, X. Zhang, S. Wang et al., "Isolation and comparison of mesenchymal stem-like cells from human gastric cancer and adjacent non-cancerous tissues," Journal of Cancer Research and Clinical Oncology, vol. 137, no. 3, pp. 495-504, 2011.

[52] H. Ponta, L. Sherman, and P. A. Herrlich, "CD44: from adhesion molecules to signalling regulators," Nature Reviews. Molecular Cell Biology, vol. 4, no. 1, pp. 33-45, 2003.

[53] F. Huang, M. Wang, T. Yang et al., "Gastric cancer-derived MSC-secreted PDGF-DD promotes gastric cancer progression," Journal of Cancer Research and Clinical Oncology, vol. 140, no. 11, pp. 1835-1848, 2014. 
[54] W. Li, Y. Zhou, J. Yang et al., "Gastric cancer-derived mesenchymal stem cells prompt gastric cancer progression through secretion of interleukin-8," Journal of Experimental \& Clinical Cancer Research, vol. 34, no. 1, p. 52, 2015.

[55] L. Sun, Q. Wang, B. Chen et al., "Human gastric cancer mesenchymal stem cell-derived IL15 contributes to tumor cell epithelial-mesenchymal transition via upregulation Tregs ratio and PD-1 expression in CD4 ${ }^{+} \mathrm{T}$ cell," Stem Cells and Development, vol. 27, no. 17, pp. 1203-1214, 2018.

[56] M. Musumeci, V. Coppola, A. Addario et al., "Control of tumor and microenvironment cross-talk by miR-15a and miR-16 in prostate cancer," Oncogene, vol. 30 , no. 41, pp. 4231-4242, 2011.

[57] M. V. Iorio and C. M. Croce, "MicroRNA dysregulation in cancer: diagnostics, monitoring and therapeutics. A comprehensive review," EMBO Molecular Medicine, vol. 4, no. 3, pp. 143-159, 2012.

[58] S. Volinia, G. A. Calin, C. G. Liu et al., "A microRNA expression signature of human solid tumors defines cancer gene targets," Proceedings of the National Academy of Sciences of the United States of America, vol. 103, no. 7, pp. 2257-2261, 2006.

[59] X. L. Yan, Y. L. Jia, L. Chen et al., "Hepatocellular carcinomaassociated mesenchymal stem cells promote hepatocarcinoma progression: role of the S100A4-miR155-SOCS1-MMP9 axis," Hepatology, vol. 57, no. 6, pp. 2274-2286, 2013.

[60] S. Y. Sung, C. H. Liao, H. P. Wu et al., "Loss of let-7 MicroRNA upregulates IL- 6 in bone marrow-derived mesenchymal stem cells triggering a reactive stromal response to prostate cancer," PLoS One, vol. 8, no. 8, article e71637, 2013.

[61] K. De Veirman, J. Wang, S. Xu et al., "Induction of miR-146a by multiple myeloma cells in mesenchymal stromal cells stimulates their pro-tumoral activity," Cancer Letters, vol. 377, no. 1, pp. 17-24, 2016.

[62] M. Wang, C. Zhao, H. Shi et al., "Deregulated microRNAs in gastric cancer tissue-derived mesenchymal stem cells: novel biomarkers and a mechanism for gastric cancer," British Journal of Cancer, vol. 110, no. 5, pp. 1199-1210, 2014.

[63] R. Ji, X. Zhang, H. Qian et al., "miR-374 mediates the malignant transformation of gastric cancer-associated mesenchymal stem cells in an experimental rat model," Oncology Reports, vol. 38, no. 3, pp. 1473-1481, 2017.

[64] Z. Pan, Y. Tian, B. Zhang et al., "YAP signaling in gastric cancer-derived mesenchymal stem cells is critical for its promoting role in cancer progression," International Journal of Oncology, vol. 51, no. 4, pp. 1055-1066, 2017.

[65] B. I. Koh and Y. Kang, "The pro-metastatic role of bone marrow-derived cells: a focus on MSCs and regulatory T cells," EMBO Reports, vol. 13, no. 5, pp. 412-422, 2012.

[66] W. Ling, J. Zhang, Z. Yuan et al., "Mesenchymal stem cells use IDO to regulate immunity in tumor microenvironment," Cancer Research, vol. 74, no. 5, pp. 1576-1587, 2014.

[67] K. Yoshikawa, S. Mitsunaga, T. Kinoshita et al., "Impact of tumor-associated macrophages on invasive ductal carcinoma of the pancreas head," Cancer Science, vol. 103, no. 11, pp. 2012-2020, 2012.

[68] P. F. Yu, Y. Huang, Y. Y. Han et al., “TNF $\alpha$-activated mesenchymal stromal cells promote breast cancer metastasis by recruiting CXCR2 ${ }^{+}$neutrophils," Oncogene, vol. 36, no. 4, pp. 482-490, 2017.
[69] B. Z. Qian, J. Li, H. Zhang et al., "CCL2 recruits inflammatory monocytes to facilitate breast-tumour metastasis," Nature, vol. 475, no. 7355, pp. 222-225, 2011.

[70] S. K. Wculek and I. Malanchi, "Neutrophils support lung colonization of metastasis-initiating breast cancer cells," Nature, vol. 528, no. 7582, pp. 413-417, 2015.

[71] Q. Zhu, X. Zhang, L. Zhang et al., "The IL-6-STAT3 axis mediates a reciprocal crosstalk between cancer-derived mesenchymal stem cells and neutrophils to synergistically prompt gastric cancer progression," Cell Death \& Disease, vol. 5, no. 6, article e1295, 2014.

[72] M. Quante, S. P. Tu, H. Tomita et al., "Bone marrow-derived myofibroblasts contribute to the mesenchymal stem cell niche and promote tumor growth," Cancer Cell, vol. 19, no. 2, pp. 257-272, 2011.

[73] Y. Shi, L. Du, L. Lin, and Y. Wang, "Tumour-associated mesenchymal stem/stromal cells: emerging therapeutic targets," Nature Reviews. Drug Discovery, vol. 16, no. 1, pp. 35-52, 2017.

[74] R. Lin, H. Ma, Z. Ding et al., "Bone marrow-derived mesenchymal stem cells favor the immunosuppressive T cells skewing in a Helicobacter pylori model of gastric cancer," Stem Cells and Development, vol. 22, no. 21, pp. 2836-2848, 2013.

[75] J. J. Montesinos, L. Mora-Garcia Mde, H. Mayani et al., "In vitro evidence of the presence of mesenchymal stromal cells in cervical cancer and their role in protecting cancer cells from cytotoxic T cell activity," Stem Cells and Development, vol. 22, no. 18, pp. 2508-2519, 2013.

[76] M. de Lourdes Mora-García, R. García-Rocha, O. MoralesRamírez et al., "Mesenchymal stromal cells derived from cervical cancer produce high amounts of adenosine to suppress cytotoxic T lymphocyte functions," Journal of Translational Medicine, vol. 14, no. 1, p. 302, 2016.

[77] M. Wang, B. Chen, X. X. Sun et al., "Gastric cancer tissuederived mesenchymal stem cells impact peripheral blood mononuclear cells via disruption of Treg/Th17 balance to promote gastric cancer progression," Experimental Cell Research, vol. 361, no. 1, pp. 19-29, 2017.

[78] L. Sun, Q. Wang, B. Chen et al., "Gastric cancer mesenchymal stem cells derived IL-8 induces PD-L1 expression in gastric cancer cells via STAT3/mTOR-c-Myc signal axis," Cell Death \& Disease, vol. 9, no. 9, p. 928, 2018.

[79] R. Xu, X. Zhao, Y. Zhao et al., "Enhanced gastric cancer growth potential of mesenchymal stem cells derived from gastric cancer tissues educated by $\mathrm{CD} 4^{+} \mathrm{T}$ cells," Cell Proliferation, vol. 51, no. 2, article e12399, 2018.

[80] X. Li, Y. Zhang, Y. Zhang, J. Ding, K. Wu, and D. Fan, "Survival prediction of gastric cancer by a seven-microRNA signature," Gut, vol. 59, no. 5, pp. 579-585, 2010.

[81] T. Ueda, S. Volinia, H. Okumura et al., "Relation between microRNA expression and progression and prognosis of gastric cancer: a microRNA expression analysis," The Lancet Oncology, vol. 11, no. 2, pp. 136-146, 2010.

[82] R. Liu, C. Zhang, Z. Hu et al., "A five-microRNA signature identified from genome-wide serum microRNA expression profiling serves as a fingerprint for gastric cancer diagnosis," European Journal of Cancer, vol. 47, no. 5, pp. 784-791, 2011.

[83] M. Tsujiura, D. Ichikawa, S. Komatsu et al., "Circulating microRNAs in plasma of patients with gastric cancers," British Journal of Cancer, vol. 102, no. 7, pp. 1174-1179, 2010.

[84] F. Huang, Y. Yao, J. Wu et al., "Curcumin inhibits gastric cancer-derived mesenchymal stem cells mediated angiogenesis 
by regulating NF- $\kappa \mathrm{B} / \mathrm{VEGF}$ signaling," American Journal of Translational Research, vol. 9, no. 12, pp. 5538-5547, 2017.

[85] Y. Zhu, B. Zhang, A. Gong et al., "Anti-cancer drug 3, 3' -diindolylmethane activates Wnt 4 signaling to enhance gastric cancer cell stemness and tumorigenesis," Oncotarget, vol. 7, no. 13, pp. 16311-16324, 2016.

[86] L. M. Cook, J. S. Frieling, N. Nerlakanti et al., "Betaglycan drives the mesenchymal stromal cell osteogenic program and prostate cancer-induced osteogenesis," Oncogene, vol. 38, no. 44, pp. 6959-6969, 2019.

[87] J. Du, A. Liu, R. Zhu et al., “The Different Effects of IFN- $\beta$ and IFN- $\gamma$ on the Tumor-Suppressive Activity of Human Amniotic Fluid-Derived Mesenchymal Stem Cells," Stem Cells International, vol. 2019, Article ID 4592701, 15 pages, 2019.

[88] R. M. Hughes, B. W. Simons, H. Khan et al., "Asporin restricts mesenchymal stromal cell differentiation, alters the tumor microenvironment, and drives metastatic progression," Cancer Research, vol. 79, no. 14, pp. 3636-3650, 2019.

[89] G. Baek, H. Choi, Y. Kim, H. C. Lee, and C. Choi, "Mesenchymal stem cell-derived extracellular vesicles as therapeutics and as a drug delivery platform," Stem Cells Translational Medicine, vol. 8, no. 9, pp. 880-886, 2019.

[90] L. Cortes-Dericks and D. Galetta, "The therapeutic potential of mesenchymal stem cells in lung cancer: benefits, risks and challenges," Cellular Oncology (Dordrecht), vol. 42, no. 6, pp. 727-738, 2019.

[91] M. Poggio, T. Hu, C. C. Pai et al., "Suppression of exosomal PD-L1 induces systemic anti-tumor immunity and memory," Cell, vol. 177, no. 2, pp. 414-427.e13, 2019.

[92] L. Gauthier, A. Morel, N. Anceriz et al., "Multifunctional natural killer cell engagers targeting NKp46 trigger protective tumor immunity," Cell, vol. 177, no. 7, pp. 1701-1713.e16, 2019.

[93] J. T. Neal, X. Li, J. Zhu et al., "Organoid modeling of the tumor immune microenvironment," Cell, vol. 175, no. 7, pp. 19721988.e16, 2018.

[94] W. Sheng, M. W. LaFleur, T. H. Nguyen et al., "LSD1 ablation stimulates anti-tumor immunity and enables checkpoint blockade," Cell, vol. 174, no. 3, pp. 549-563.e19, 2018.

[95] G. Dewson and J. Silke, "The walrus and the carpenter: complex regulation of tumor immunity in colorectal cancer," Cell, vol. 174, no. 1, pp. 14-16, 2018.

[96] Y. Dong, Q. Sun, and X. Zhang, "PD-1 and its ligands are important immune checkpoints in cancer," Oncotarget, vol. 8, no. 2, pp. 2171-2186, 2017.

[97] Z. Pan, Y. Tian, C. Cao, and G. Niu, "The emerging role of YAP/TAZ in tumor immunity," Molecular Cancer Research, vol. 17, no. 9, pp. 1777-1786, 2019.

[98] W. Zhu, L. Huang, Y. Li et al., "Mesenchymal stem cellsecreted soluble signaling molecules potentiate tumor growth," Cell Cycle, vol. 10, no. 18, pp. 3198-3207, 2011. 


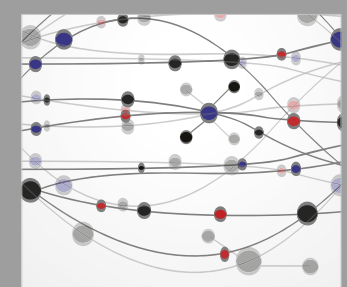

The Scientific World Journal
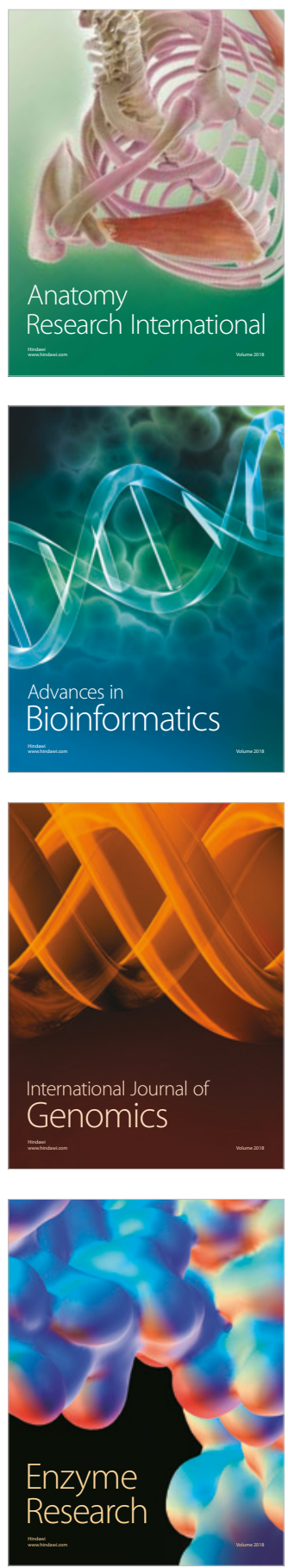
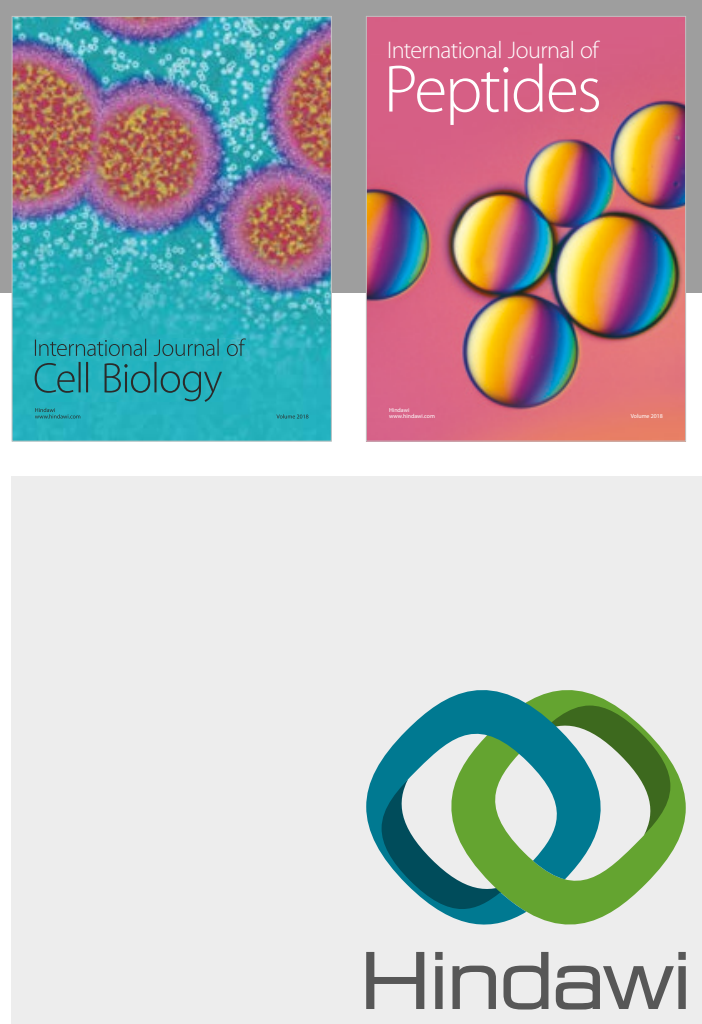

Submit your manuscripts at

www.hindawi.com
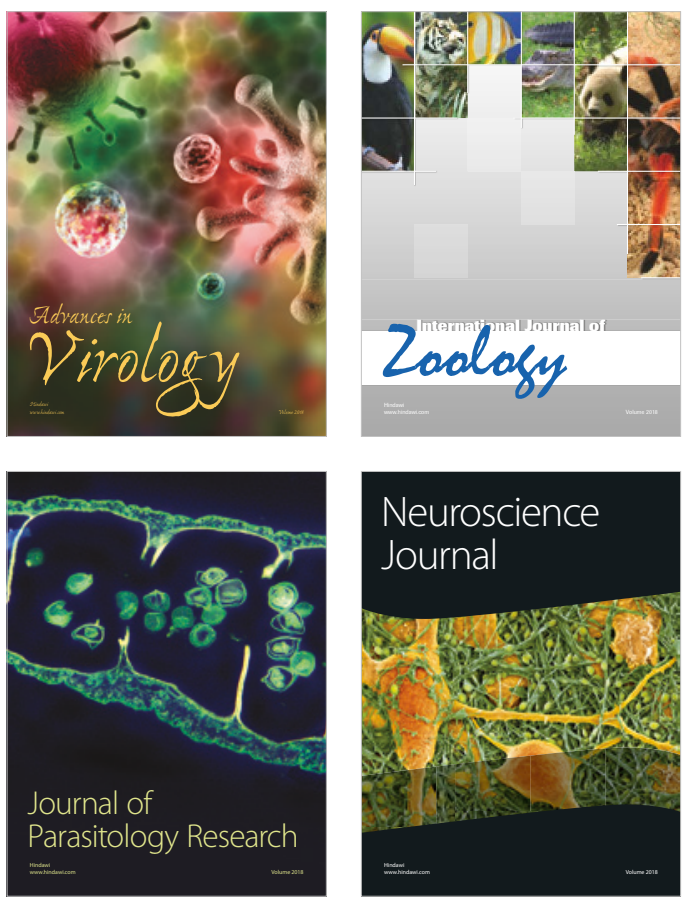
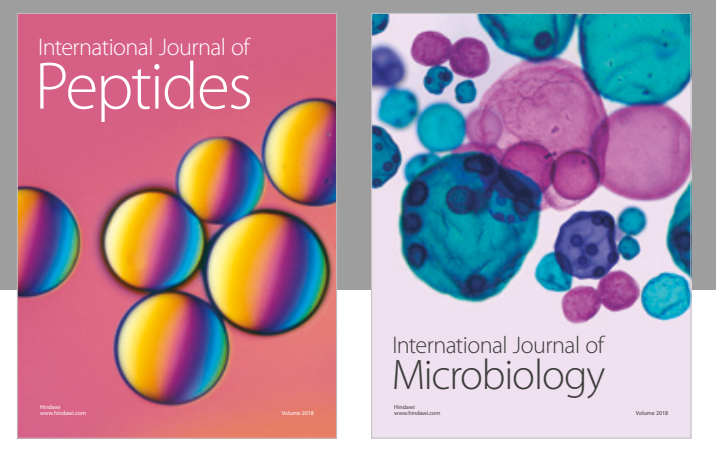

nternational Journal of Microbiology
Journal of
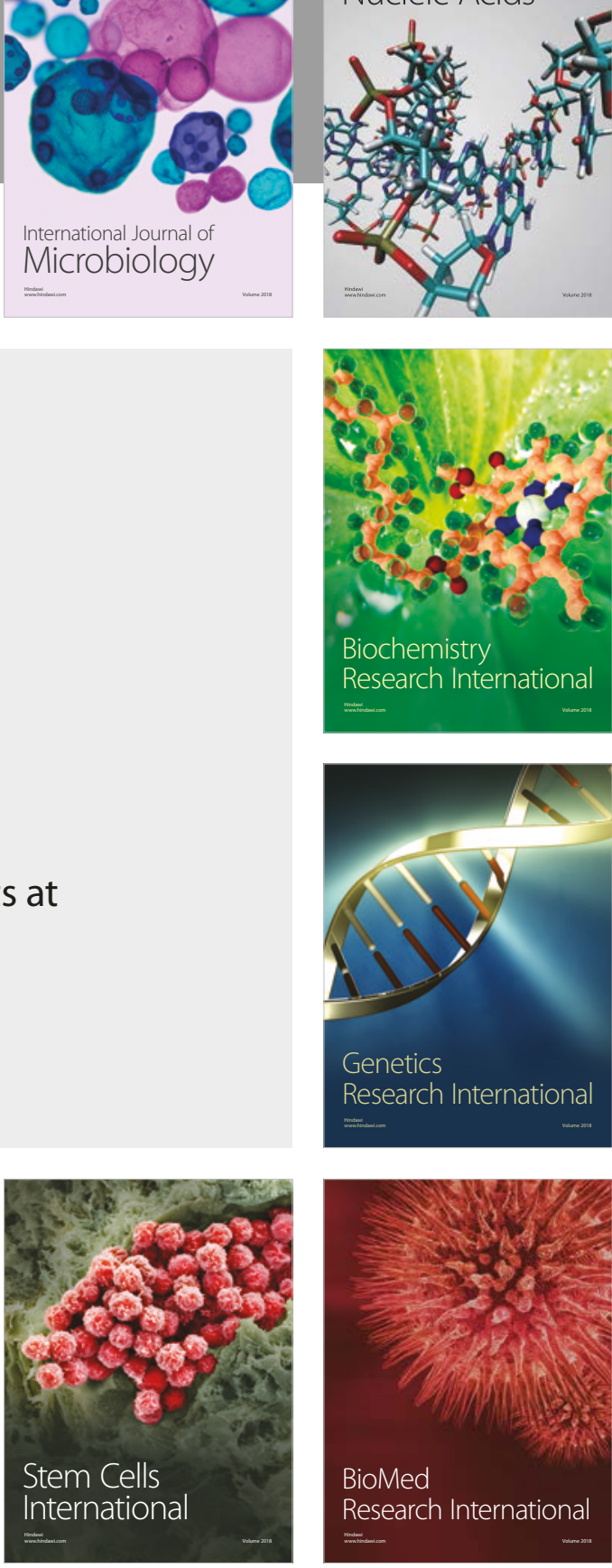
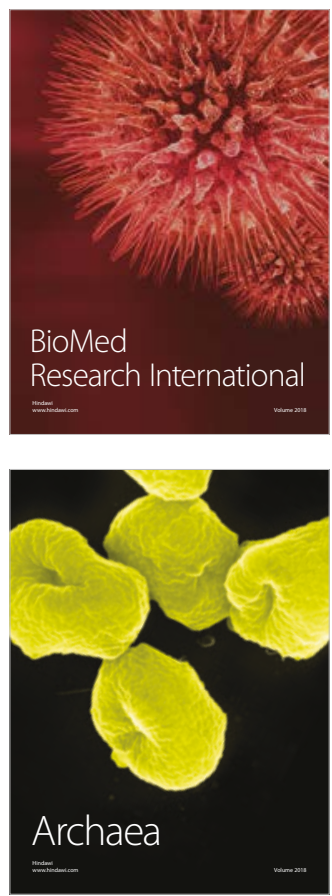